\title{
Leptospirose humana no município de Belo Horizonte, Minas Gerais, Brasil: uma abordagem geográfica
}

\author{
Human leptospirosis in Belo Horizonte City, Brazil: a geographic approach
}

\begin{abstract}
Cláudia Maria de Figueiredo', Ana Clara Mourão', Marluce Aparecida A. de Oliveira², Willian Rosa Alves', Márcia Costa Ooteman ${ }^{2}$, Chequer Buffe Chamone ${ }^{3}$ e Matilde Cota Koury ${ }^{2}$
\end{abstract}

\begin{abstract}
Resumo Uma análise retrospectiva da leptospirose humana no município de Belo Horizonte em 1995 foi realizada usando recursos de geoprocessamento. Trinta casos suspeitos e 19 casos confirmados foram localizados na área. A predominância de casos confirmados foi registrada nas regiões norte, nordeste e oeste $(68,4 \pm 13 \%)$. Nas áreas de favelas e bolsões de pobreza foram identificados os principais focos da doença, $73,7 \pm 12 \%$ dos casos confirmados e $26,7 \pm 12 \%$ dos casos suspeitos. Na periferia, onde ocorreu um aumento populacional, localizaram-se $95 \pm 6 \%$ dos casos confirmados, sendo o local com maior carência de infraestrutura básica. Na distribuição espacial dos principais cursos d'água do município, observou-se que $50 \pm$ $14 \%$ dos casos suspeitos e $42 \pm 14 \%$ dos casos confirmados estavam localizados nas áreas com maior concentração de redes fluviais. Casos suspeitos (83,3 $\pm 10 \%)$ e confirmados $(79 \pm 11 \%)$ ocorreram em partes altimétricas mais baixas da cidade (750 a 1.000m) e $78 \pm 12 \%$ dos indivíduos tiveram contato com água e / ou animais contaminados.
\end{abstract}

Palavras-chaves: Leptospirose. Reação de soroaglutinação microscópica. Geoprocessamento.

\begin{abstract}
A retrospective study on human leptospirosis, was done in Belo Horizonte in 1995, using geographic processing resources. Thirty suspected and 19 confirmed cases of leptospirosis were mapped in the area. The majority of confirmed cases $(68.4 \pm 13 \%)$ were located in North, Northeast and West of the city. The main foci of disease were found in slums and other poor areas: $73.7 \pm 12 \%$ of the confirmed cases and $26.7 \pm 12 \%$ of suspected cases. Ninety-five percent $\pm 6 \%$ of the confirmed cases were found in the outskirts of the city where there was a population increase and inadequate infrastructure. It was observed that $50 \pm 14 \%$ of the suspected cases and $42 \pm 14 \%$ of the confirmed cases were found in areas of high concentration of water resources. Suspected (83.3 $\pm 10 \%)$ and confirmed cases $(79 \pm 11 \%)$ occurred in lower altitude areas of the city (750 to $1,000 \mathrm{~m})$ and $78 \pm 12 \%$ of the individuals had been in contact with contaminated water and/or animals.
\end{abstract}

Key-words: Leptospirosis. Microscopic agglutination test. Geographic processing.

A leptospirose é uma doença infecciosa sistêmica, aguda, febril, causada por espiroquetas do gênero Leptospira. No Brasil, a leptospirose é considerada uma doença endêmica e constitui um sério risco à saúde pública.

A manutenção de leptospira nas regiões urbanas e rurais do Brasil é favorecida pelo clima tropical úmido e uma vasta população de roedores. O crescimento urbano desordenado e a grande quantidade de lixo espalhado sobre vias e terrenos baldios propiciam também um ambiente ideal para a proliferação da população murina.

Trabalhos epidemiológicos sobre leptospirose foram realizados no Brasil' 231012151617 . Em São Paulo, no Instituto de Infectologia Emilio Ribas, 43 crianças foram hospitalizadas com a doença no período entre janeiro de 1989 e dezembro de 1995 , e $88 \%$ tiveram contato com água contaminada ${ }^{12}$. Em 152 municípios de São Paulo foram estudados 2.969 casos de leptospirose, no período entre janeiro de 1989 e dezembro de 1994, verificando com maior freqüência coeficientes entre 2 e 10 casos $/ 100.000$ habitantes $^{15}$. Dos sorovares de leptospiras isolados de pacientes no período de 1986 a 1989, em São Paulo, 14 das amostras sorotipadas eram do sorovar copenhagenit ${ }^{4}$. No Rio de Janeiro, os primeiros surtos epidêmicos ocorreram a partir da década de 1960 e sempre coincidiram com as inundações após temporais de verão ${ }^{7}$. De 1970 a 1982 , 884 casos foram diagnosticados na Fundação Oswaldo Cruz ${ }^{1}$. De janeiro de 1983 a junho de 1998, 188 soros

\footnotetext{
1. Departamento de Geografia do Instituto de Geociências da Universidade Federal de Minas Gerais. 2. Departamento de Microbiologia do Instituto de Ciências Biológicas da Universidade Federal de Minas Gerais. 3. Divisão de Biologia Médica do Instituto Octávio Magalhães da Fundação Ezequiel Dias, Belo Horizonte, MG.

Endereço para correspondência: Dra. Matilde Cota Koury. Deptoำ de Microbiologia/ICB/UFMG. Av. Antônio Carlos 6627, Caixa Postal 486, 31270-901 Belo Horizonte, MG.

Fone: 5531 499-2764 Fax: 5531 499-2733

E-mail:kourymat@mono.icb.ufmg.br

Recebido para publicação em 8/9/2000.
} 
de crianças foram examinados pela reação de soroaglutinação microscópica ( SAM ) e 27,6\% foram positivos $^{3}$.

Em Salvador, de 326 casos com suspeita de leptospirose, no período entre março e novembro de 1996, 133 foram confirmados laboratorialmente e 60 foram considerados casos suspeitos. O sorovar copenhageni foi isolado em $87 \%$ dos casos que tiveram cultura de sangue positiva ${ }^{10}$.

Em Minas Gerais poucos trabalhos epidemiológicos foram realizados. A Fundação Ezequiel Dias, laboratório de referência do estado para o diagnóstico das leptospiroses, recebeu 411 amostras de sangue de pacientes suspeitos no período de 1988 a 1993, que foram examinados pela reação de SAM obtendo-se $28,5 \%$ de positividade ${ }^{14}$. Em Uberaba, os indivíduos foram separados em dois grupos, os que tinham maior contato com animais (257) e os com menor contato (188). Os soros de $46,7 \%$ dos indivíduos do primeiro grupo e 19,3\% do segundo apresentaram-se positivos na reação de SAM ${ }^{17}$.

Embora haja registro sistemático de casos de leptospirose na literatura médica de quase todo os países, existe uma lacuna de conhecimento entre a incidência da doença e aspectos geográficos. Esse trabalho tem como objetivos demonstrar, na dimensão espacial, a ocorrência da leptospirose no município de Belo Horizonte, Minas Gerais e fornecer subsídios para futuras pesquisas e adoção de medidas preventivas por parte das autoridades sanitárias do município. O expediente de analisar os soros de pacientes, com suspeita clínica de leptospirose, encaminhados á FUNED para diagnóstico se constituiu em uma ferramenta inicial para a localização dos focos de leptospirose no município. A leptospirose foi estudada a partir de uma perspectiva geográfica e as alterações do equilíbrio homem-ambiente foram identificadas a partir de transformações produtivas territoriais e espaciais que a ordem social impõe sobre o espaço. Relacionaram-se os aspectos físicos e sociais, embutidos nesse espaço urbano, que propiciaram o aparecimento da leptospirose. Os casos suspeitos e confirmados foram localizados no Município, considerando o local de moradia dos indivíduos e suas relações com as fontes de contágio, o crescimento populacional, a presença de favelas e bolsões de pobreza, a altimetria, os principais cursos de água e ausência de redes de esgoto.

\section{MATERIAL E MÉTODOS}

Seleção de casos. A Fundação Ezequiel Dias (FUNED), Laboratório de referência para diagnóstico de leptospirose no Estado de Minas Gerais, recebeu 162 soros de pacientes com suspeita da doença no ano de 1995. As amostras eram acompanhadas de fichas epidemiológicas, preenchidas nos diferentes hospitais da rede pública e privada e, também, nas diferentes regionais, contendo informações sobre os pacientes, como idade, sexo, local de moradia, contato com animais e água.

Dos 162 soros recebidos de pacientes com suspeita da doença, 49 (30,2\%) foram do município de Belo Horizonte e $113(69,8 \%)$ de outros municípios do Estado de Minas Gerais. Para essa análise retrospectiva da leptospirose humana no município de Belo Horizonte foram examinados os 49 soros de pacientes com suspeita da doença pela reação de SAM. De acordo com o Manual de Leptospirose ${ }^{6}$, o diagnóstico de leptospirose depende da avaliação de fatores relacionados ao quadro clínico, aos resultados laboratoriais e aos dados epidemiológicos. Consideraram-se casos suspeitos, os indivíduos com antecedentes epidemiológicos e os que apresentaram sinais e sintomas sugestivos da doença, como febre, mialgias, vômitos, calafrios, conjuntivite, icterícia, hemorragias, alterações hepáticas, renais e vasculares e casos confirmados, os que apresentaram resultados laboratoriais positivos, SAM com soro conversão (aumento do título de quatro vezes ou mais) ou título igual ou maior que 800 na reação de SAM.

Reação de soroaglutinação microscópica. A reação de $\mathrm{SAM}^{4}$ foi realizada utilizando como antígeno 22 sorovares de leptospiras vivas cultivadas em meio de Ellinghausen e McCullough, modificado ${ }^{9}$. Os seguintes sorovares foram usados como antígenos: australis, autumnalis, andamana, bataviae, bratislava, butembo, canicola, castellonis, celledoni, djasiman, fort bragg, grippotyphosa, hardjo, hebdomadis, icterohaemorrhagiae, javanica, naam, patoc, panama, pyrogenis, pomona e wolffi. O título aglutinante foi considerado como o inverso da maior diluição do soro que $50 \%$ das leptospiras apresentaram aglutinadas.

Mapeamento da doença. O local de mapeamento da doença foi o município de Belo Horizonte, situado na zona metalúrgica da estado de Minas Gerais, com área correspondente à $335 \mathrm{Km}^{2}$ e dividido em nove Administrações Regionais (Barreiro, Oeste, CentroSul, Leste, Noroeste, Pampulha, Venda Nova, Norte e Nordeste) ${ }^{13}$, que serviram como guia na localização dos casos suspeitos e confirmados da doença. Para o mapeamento utilizaram-se os dados contidos nas fichas epidemiológicas.

Cartografia digital. A cartografia digital é um recurso de geoprocessamento que permite a elaboração de banco de dados cartográficos para construção de cartas temáticas. A elaboração das cartas temáticas foi realizada a partir de uma base cartográfica digital comum a todos os temas trabalhados. Essa base foi obtida em um mapa da Prodabel, contendo os seguintes dados: limites de Belo Horizonte, divisões administrativas governamentais e a Lagoa da Pampulha ${ }^{13}$. Os dados foram digitalizados e localizados naquela base em sistema de coordenadas "Universal Transversal Mercator" (UTM), cuja projeção X/Y é 
ortogonal, dada em $\mathrm{km} / \mathrm{m}$. Os temas analisados e mapeados foram os bolsões de pobreza, áreas não servidas por rede de esgoto, crescimento populacional por sub-região, cursos de águas principais e altimetria $^{73}$. A ocorrência de casos confirmados e suspeitos em cada área temática foi determinada percentualmente, em relação ao número total de casos recebidos $(n=49)$. Assim sendo, cada valor obtido de ocorrência $(p)$ foi descrito utilizando-se o intervalo de confiança.

\section{RESULTADOS}

Dos 49 soros examinados pela reação de SAM, $30(61,2 \%)$ apresentaram resultados negativos com títulos iguais ou menores que 200. Esses casos foram considerados como suspeitos com base na avaliação do quadro clínico e dados epidemiológicos e não foi possível obter a segunda amostra de soro para confirmação da doença. Dezenove $(38,8 \%)$ soros apresentaram resultados positivos na reação de SAM com títulos iguais ou maiores que 800 na primeira amostra do soro, ou soro conversão (aumento do título de quatro vezes ou mais). O título mais elevado foi para o sorovar icterohaemorrhagiae (Tabela 1).

Tabela 1 - Títulos obtidos na reacão de soroaglutinação microscópica para diferentes sorovares de leptospira em soros de pacientes ( $n=19)$, casos confirmados, no Município de Belo Horizonte, no ano de 1995.

\begin{tabular}{|c|c|c|c|c|c|c|c|c|c|c|c|c|c|c|c|c|c|c|c|}
\hline \multirow[t]{2}{*}{ Sorovares de leptospira } & \multicolumn{19}{|c|}{ Soros de pacientes } \\
\hline & 1 & 2 & 3 & 4 & 5 & 6 & $7^{*}$ & $8^{*}$ & 9 & 10 & 11 & 12 & $13^{*}$ & 14 & $15^{*}$ & 16 & 17 & 18 & 19 \\
\hline patoc & & 100 & 400 & & 200 & 200 & & & & 400 & & & & & 200 & & & & \\
\hline djasiman & & & & 800 & 400 & 200 & 200 & & & & & & & & & & & & \\
\hline autumnalis & & & & 200 & & 400 & & & & & & 800 & & & 100 & 800 & & & 800 \\
\hline andamana & & & 400 & 800 & 400 & & 800 & 100 & 200 & 200 & & & & & & & & & \\
\hline bataviae & & & & 400 & & & & & & & 200 & & & & & & 100 & & 800 \\
\hline bratislava & & & & & & & & 200 & 200 & 400 & 200 & 200 & & & & & 100 & & \\
\hline butembo & & & & & & & & & & & & & & & & 200 & 100 & & \\
\hline canicola & & & & 200 & & & & 100 & & 1600 & 100 & 400 & & 800 & & & & 800 & \\
\hline castellonis & 100 & 100 & & & & & & & & 100 & & & & & 100 & & & & \\
\hline celledoni & & & & & & & & & & & & & & & 200 & & & & \\
\hline icterohaemorrhagiae & 800 & 800 & 4001 & 1600 & 800 & 1600 & & 1600 & 800 & 3200 & 800 & 800 & 800 & & 800 & & 800 & 200 & 800 \\
\hline javanica & & 100 & 200 & & 400 & 200 & & & & & & & & & & & & & \\
\hline hebdomadis & 400 & & & & & & & & & & & & 100 & 100 & & & 200 & 400 & 200 \\
\hline wolffi & & & 400 & & & & & 400 & & 400 & & & & & & & & & \\
\hline fort-bragg & & & 400 & 100 & & & & 200 & 200 & 400 & & 400 & 200 & & 400 & & 200 & & \\
\hline grippotyphosa & & & & & & & & 100 & & & & & & & & & & & \\
\hline hardjo & & & & & & & & & & & & & & 200 & & & & & \\
\hline naan & 800 & 800 & 800 & & & & & 400 & & 800 & 400 & & & & & & 800 & 400 & \\
\hline pomona & & & & & & & & & & & & & & & 100 & & & & \\
\hline australis & & & & & & & & & & & & & & & 100 & & & & \\
\hline pomona & & & & & & & & & & & & & & 100 & & & & & \\
\hline pyrogenis & & & & & & & 100 & & & & & & & & & & & & \\
\hline
\end{tabular}

* soros pareados

A Tabela 2 mostra que a faixa etária variou de 2 a 89 anos, e que a doença atingiu $73,7 \%$ dos pacientes com faixa etária de 10 aos 40 anos. Em relação ao sexo, a doença ocorreu em $89,5 \%$ dos pacientes do sexo masculino. Dos 30 casos suspeitos de leptospirose, $7(23,3 \%)$ indivíduos tiveram contato somente com água, 9 (30\%) somente com animais, 4 (13,3\%) com animais e água, $2(6,7 \%)$ relataram viagem a zona rural e presença de terrenos baldios próximo à moradia e
$8(26,7 \%)$ não informaram. Dos 19 casos confirmados, $9(47,4 \%)$ dos indivíduos tiveram contato somente com água, 3 (15,8\%) somente com animais, $6(31,6 \%) \mathrm{com}$ água e animais e 1 (5,2\%) não informou.

A Figura 1 mostra a localização dos casos suspeitos (30) e confirmados (19) de leptospirose e sua relação com as fontes de contágio, no município de Belo Horizonte, no ano de 1995. A predominância de casos confirmados foi registrada nas regiões norte, nordeste 
Tabela 2 - Distribuição de casos suspeitos $(n=30)$ e confirmados $(n=19)$ de leptospirose humana no Município de Belo Horizonte, no ano de 1995 e sua relação com as fontes de contágio.

\begin{tabular}{|c|c|c|c|c|c|c|c|c|}
\hline \multirow{2}{*}{$\begin{array}{l}\text { Casos suspeitos } \\
\text { e confirmados* }\end{array}$} & \multirow{2}{*}{$\begin{array}{r}\text { Idade } \\
\text { (anos) }\end{array}$} & \multirow{2}{*}{$\begin{array}{r}\text { Sexo } \\
M / F\end{array}$} & \multirow[b]{2}{*}{ Região de moradia } & \multicolumn{4}{|c|}{ Contato } & \multirow{2}{*}{$\begin{array}{l}\operatorname{SAM}_{+/-}^{* *} \\
+\end{array}$} \\
\hline & & & & animal & água & s/dados & outros & \\
\hline$\overline{01^{*}}$ & 13 & $M$ & Barreiro & $x$ & $x$ & & & + \\
\hline $02^{*}$ & 49 & $M$ & Nordeste & & $x$ & & & + \\
\hline $03^{*}$ & 23 & M & Oeste & $\mathrm{x}$ & $x$ & & & + \\
\hline $04^{*}$ & 36 & M & Norte & & $\mathrm{x}$ & & & + \\
\hline $05^{*}$ & 26 & $\mathrm{~F}$ & Norte & $x$ & $x$ & & & + \\
\hline $06^{*}$ & 48 & M & Oeste & & $x$ & & & + \\
\hline $07^{\star}$ & 18 & M & Leste & & $x$ & & & + \\
\hline $08^{*}$ & 38 & M & Venda Nova & $x$ & $x$ & & & + \\
\hline $09^{*}$ & 21 & M & Venda Nova & & $\mathrm{x}$ & & & + \\
\hline $10^{*}$ & 38 & $M$ & Nordeste & & $x$ & & & + \\
\hline $11^{*}$ & 45 & $M$ & Oeste & $x$ & & & & + \\
\hline $12^{*}$ & 26 & $M$ & Oeste & $x$ & & & & + \\
\hline $13^{\star}$ & 16 & M & Leste & & $\mathrm{x}$ & & & + \\
\hline $14^{\star}$ & 26 & M & Venda Nova & & & $\mathrm{x}$ & & + \\
\hline $15^{*}$ & 20 & M & Nordeste & $x$ & $x$ & & & + \\
\hline $16^{*}$ & 30 & $M$ & Norte & $x$ & $x$ & & & + \\
\hline $17^{*}$ & 54 & $M$ & Oeste & & $x$ & & & + \\
\hline $18^{*}$ & 46 & $\mathrm{~F}$ & Nordeste & $x$ & & & & + \\
\hline $19^{*}$ & 36 & M & Norte & & $x$ & & & + \\
\hline 20 & 67 & $\mathrm{~F}$ & Oeste & & & $x$ & & - \\
\hline 21 & 30 & $M$ & Leste & $x$ & & & & - \\
\hline 22 & 37 & $M$ & Centro-Sul & & $x$ & & & - \\
\hline 23 & 06 & $\mathrm{~F}$ & Venda Nova & $x$ & & & & - \\
\hline 24 & 57 & $\mathrm{~F}$ & Leste & & & $\mathrm{x}$ & & - \\
\hline 25 & 14 & $M$ & Centro-Sul & $x$ & & & & - \\
\hline 26 & 43 & $M$ & Leste & & & $x$ & & - \\
\hline 27 & 20 & $M$ & Oeste & & & $x$ & & - \\
\hline 28 & 36 & M & Centro-Sul & & & $x$ & & - \\
\hline 29 & 8 & $\mathrm{~F}$ & Leste & $x$ & $x$ & & & - \\
\hline 30 & 3 & M & Noroeste & $x$ & & & & - \\
\hline 31 & 79 & $M$ & Oeste & $x$ & & & & - \\
\hline 32 & 25 & $M$ & Leste & $x$ & & & & - \\
\hline 33 & 15 & $\mathrm{~F}$ & Leste & & & & $x$ & - \\
\hline 34 & 37 & $M$ & Centro-Sul & & $x$ & & & - \\
\hline 35 & 53 & M & Centro-Sul & $x$ & & & & - \\
\hline 36 & 2 & $M$ & Noroeste & $x$ & & & & - \\
\hline 37 & 36 & $\mathrm{~F}$ & Barreiro & $x$ & $x$ & & & - \\
\hline 38 & 31 & M & Barreiro & & $x$ & & & - \\
\hline 39 & 39 & $M$ & Nordeste & & & $x$ & & - \\
\hline 40 & 40 & M & Oeste & $x$ & $x$ & & & - \\
\hline 41 & 20 & $M$ & Nordeste & & $x$ & & & - \\
\hline 42 & 89 & $M$ & Leste & & $x$ & & & - \\
\hline 43 & 15 & $M$ & Oeste & $x$ & $x$ & & & - \\
\hline 44 & 38 & $\mathrm{~F}$ & Norte & $x$ & & & & - \\
\hline 45 & 60 & $M$ & Leste & & & $x$ & & - \\
\hline 46 & 23 & $\mathrm{~F}$ & Nordeste & & $x$ & & & - \\
\hline 47 & 23 & $\mathrm{~F}$ & Nordeste & & $x$ & & & - \\
\hline 48 & - & $M$ & Leste & & & & $x$ & - \\
\hline 49 & 34 & $\mathrm{~F}$ & Centro-Sul & & & $x$ & & - \\
\hline
\end{tabular}




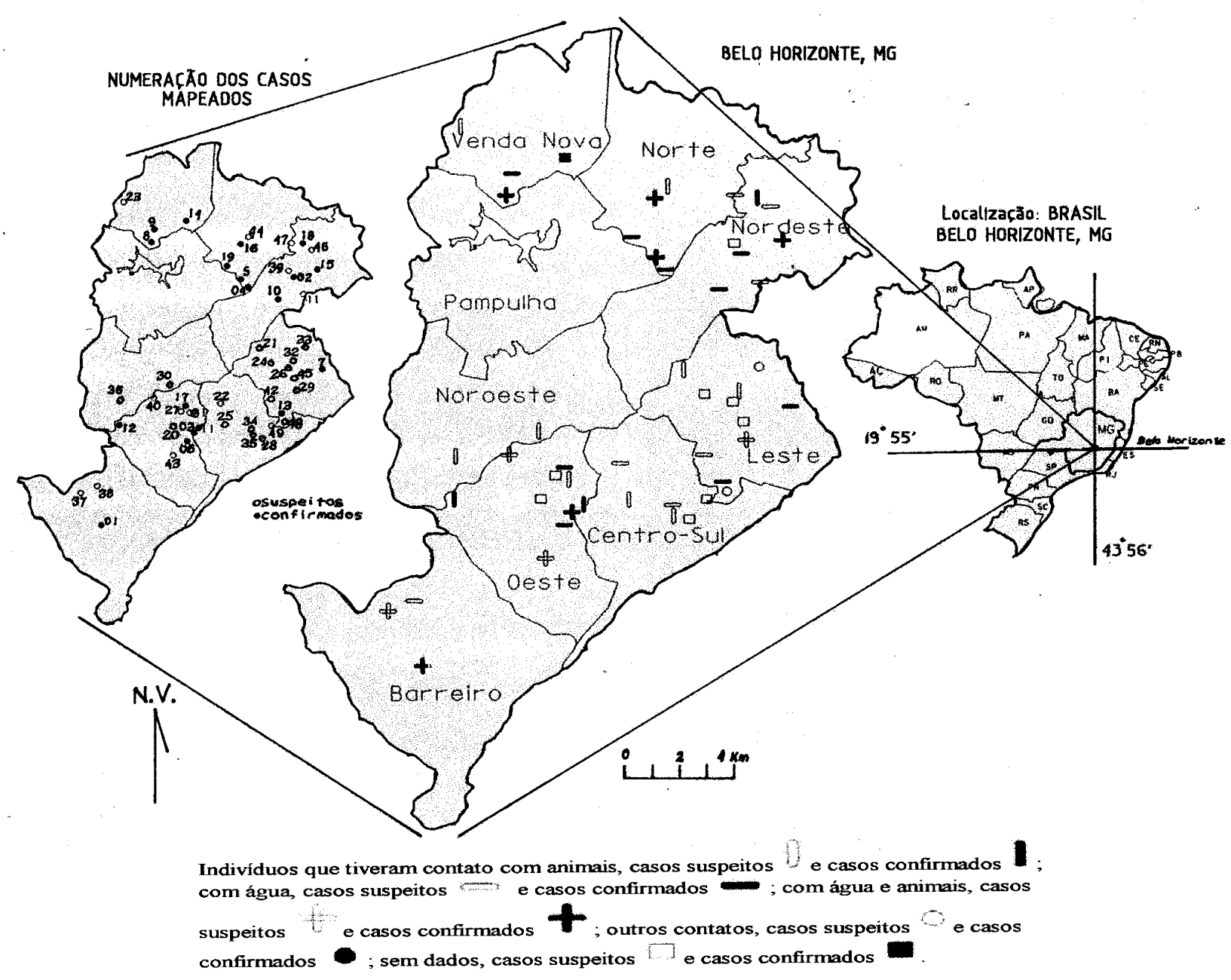

Figura 1 - Localização de casos suspeitos e confirmados de leptospirose humana e suas relações com as fontes de contágio no municipio de Belo Horizonte, MG, no ano de 1995.

e oeste de Belo Horizonte, $68,4 \pm 13 \%$. As fontes de contágio, quando localizadas em carta temática, mostram distribuição homogênea por toda a área. Do total de casos que informaram, $78 \pm 12 \%$ dos indivíduos tiveram contacto com água e/ou animais.

Nas áreas de favelas e bolsões de pobreza foram identificados os principais focos da doença, $73,7 \pm 12 \%$ dos casos confirmados e $26,7 \pm 12 \%$ dos suspeitos. A Pampulha foi a única regional que não apresentou casos de leptospirose. Na periferia, onde ocorreu um aumento populacional, localizaram-se $95 \pm 6 \%$ dos casos confirmados, sendo o local que apresenta a maior carência de infra-estrutura básica. $\mathrm{Na}$ faixa central do município observaram-se $53 \pm 14 \%$ dos casos suspeitos e $5 \pm 6 \%$ dos confirmados (Figura 2).
A distribuição espacial das áreas não atendidas por esgoto mostra que $26,3 \pm 12 \%$ dos casos confirmados estão localizados em áreas com carência de saneamento básico e 52,6 $\pm 14 \%$ são adjacentes a áreas mapeadas com ausência de rede de esgoto. $\mathrm{Na}$ distribuição espacial dos principais cursos d'água de Belo Horizonte, observa-se que $50 \pm 14 \%$ dos casos suspeitos e $42 \pm 14 \%$ dos confirmados estão localizados nas áreas com maior concentração de redes fluviais. Cerca de 53,1 $\pm 14 \%$ dos indivíduos tiveram alguma modalidade de contato hídrico, sob forma de inundações ou práticas recreativas como a natação em lagoas e riachos. Casos suspeitos $(83,3 \pm 10 \%)$ e confirmados $(79 \pm 11 \%)$ ocorreram em partes altimétricas mais baixas da cidade (750 a 1.000m) (Figura 3). 


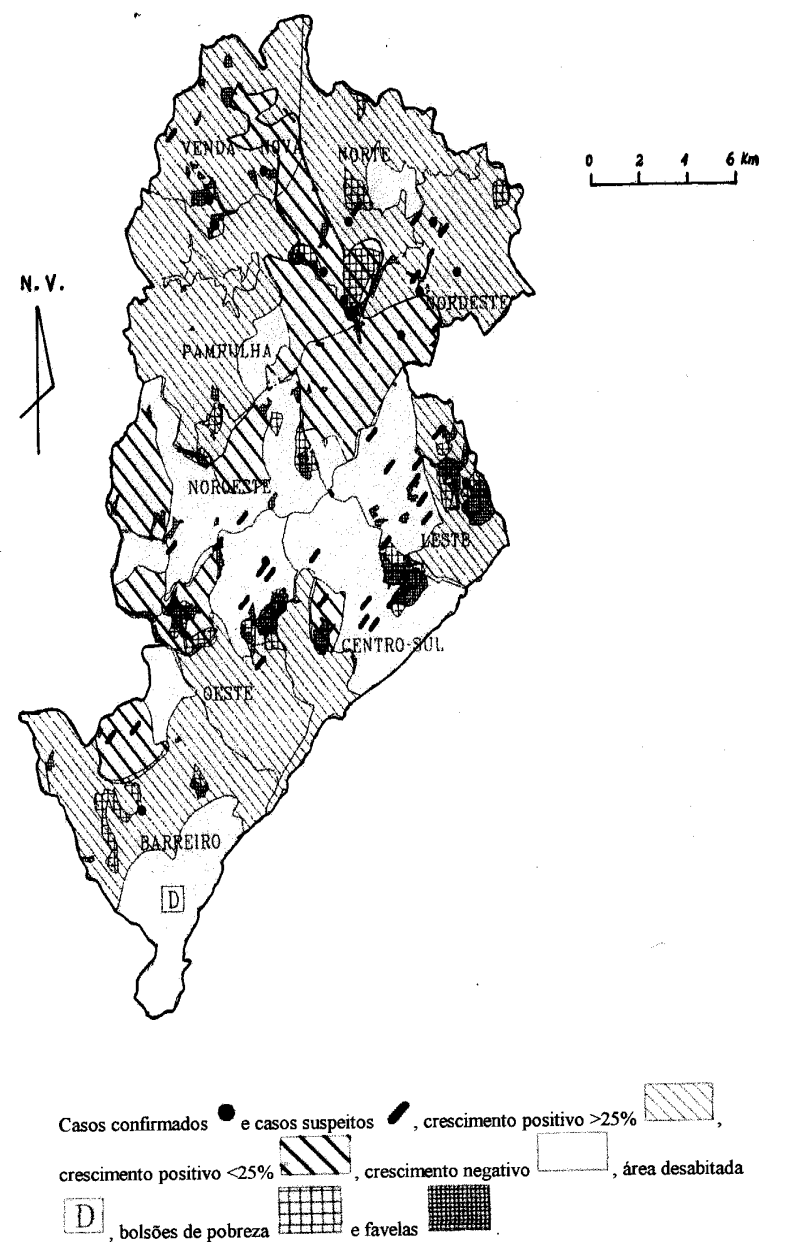

Figura 2 - Localização de casos suspeitos e confirmados de leptospirose e suas relacões com o crescimento populacional, a presença de favelas e bolsões de porbreza, no município de Belo Horizonte, MG, no ano de 1995.
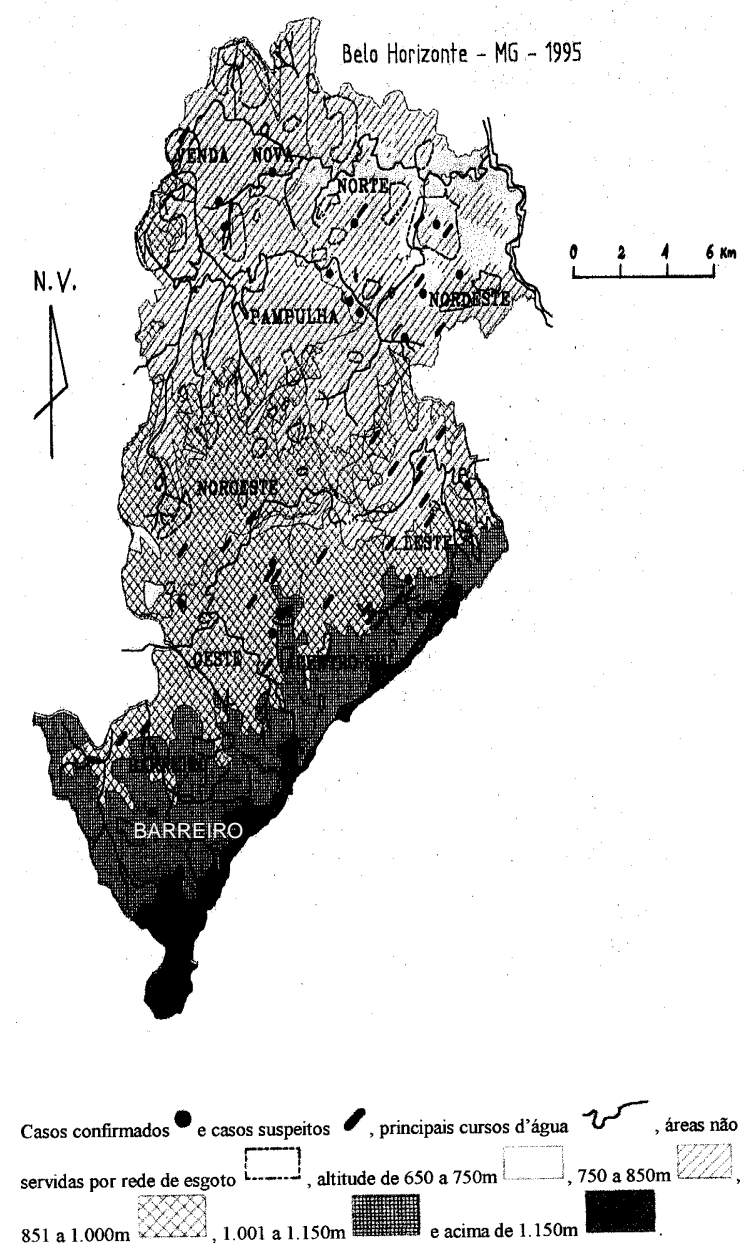

Figura 3 - Localização de casos suspeitos e confirmados de leptospirose e suas relacões com a altimetria os principais cursos d'água e a ausência de redes de esgoto, no município de Belo Horizonte, MG, no ano de 1995.

\section{DISCUSSÃO}

Esse estudo não visou estabelecer a prevalência da leptospirose em Belo Horizonte, posto que apenas os soros de pacientes sob suspeita e encaminhados para FUNED foram examinados. Em termos de saúde pública, e considerando que a leptospirose é uma doença infecciosa emergente que está espalhando do seu ambiente rural para centros urbanos, o conhecimento das regiões onde a doença ocorreu mais freqüentemente poderá não só subsidiar políticas de saneamento, mas também dar suporte a futuros trabalhos de levantamentos, para estudo de prevalência. Assim sendo, a distribuição de frequência da doença nas regiões do município de Belo Horizonte, foi definida dentro da amostra total recebida (não aleatória), com seus valores definidos por seus intervalos de confiança.
A FUNED recebeu 162, 272, 463, 216 e 593 amostras de soro de pacientes do Estado de Minas Gerais para realização de SAM para diagnóstico de leptospirose nos anos de 1995, 1996, 1997, 1998 e 1999, respectivamente. Realizamos estudo retrospectivo referente ao ano de 1995, e dos 162 soros recebidos, 49 foram provenientes do município de Belo Horizonte. Dos 49 soros de pacientes, 19 (38,8\%) apresentaram resultados positivos na reação de SAM e foram considerados casos confirmados da doença e os $30(61,2 \%)$ soros que apresentaram resultados negativos na reação de SAM foram considerados como casos suspeitos com base na avaliação do quadro clínico e dados epidemiológicos.

$\mathrm{Na}$ cidade de Salvador em 1996, ocorreu uma epidemia urbana de leptospirose, e de 193 (59\%) casos, 
$133(68,9 \%)$ foram confirmados pela reação de SAM e $60(31,1 \%)$ foram considerados casos prováveis da doença.

Dos casos confirmados, em relação ao sexo, a doença ocorreu em $89,5 \%$ dos pacientes do sexo masculino. Não existe diferença de suscetibidade quando ambos os sexos estão igualmente expostos à fonte de contágio, porém a doença ocorre predominantemente no sexo masculino ${ }^{11}$. Em relação à faixa etária de 10 aos 40 anos, a doença atingiu 73,7\% dos pacientes. A leptospirose atinge com maior freqüência os adultos jovens, dos 10 aos 39 anos de idade, sendo maior entre os de 20 e 29 anos $^{11}$.

Entre as variáveis selecionadas e mapeadas para o estudo de casos confirmados de leptospirose, as favelas e os bolsões de pobreza, foram os locais onde se identificaram mais freqüentemente a doença $(73,7 \pm 12 \%)$. Nessas áreas, onde os serviços de limpeza urbana são precários, observou-se a maior vulnerabilidade da população em contrair a doença. $A$ precária organização do espaço físico das favelas, com a ocupação desordenada e falta de saneamento básico adequado constitui potencial de risco de contaminação do ambiente com leptospiras. $\mathrm{Na}$ análise das fontes de contágio (água, animais, terrenos baldios e outros) com leptospiras verificou-se que se distribuem de forma homogênea por toda a àrea estudada. $\mathrm{Na}$ regional Pampulha, as favelas representam um fator irrelevante em termos de extensão física. As pequenas áreas de favelas dispersas na Pampulha e a não observação de casos nessa região, demonstram mais uma vez, o papel que a favela representa em termos da distribuição espacial da doença (Figura 2).

A análise da distribuição espacial das áreas não atendidas por esgoto mostra 26,3 $\pm 12 \%$ de casos confirmados inseridos na área com carência de saneamento básico e os $52,6 \pm 14 \%$ mantêm proximidade física com áreas mapeadas com falta de serviço básico de esgoto. Grande parte dos casos confirmados mostram uma estreita correlação com a falta de serviços de esgoto e a ocorrência de favelas.

Com exceção de um foco concentrado em Venda Nova, a predominância de casos confirmados foi registrada nas regionais norte, nordeste e oeste de Belo Horizonte (68,4 $\pm 13 \%)$, devido à expansão e crescimento rápido das periferias urbanas de Belo Horizonte nessa direção, sem as condições adequadas de infra-estrutura sanitária, urbanização e coleta de lixo. Nas periferias de Belo Horizonte observa-se um aumento da população que subsiste com carência de infra-estrutura básica refletida na falta de redes de esgoto e de pavimentação de vias, além de deficiência no sistema de coleta e destinação do lixo, justamente onde foram verificados $95 \pm 6 \%$ dos casos confirmados de leptospirose.

A população urbana de Belo Horizonte cresceu de maneira acelerada nas últimas décadas, substancialmente em função da modernização agrícola ocorrida no campo. Os espaços físicos da metrópole e adjacências passaram a ser transformados em função do lucro imobiliário com implicações diretas sobre as características urbanas.

Mudanças sem planejamento adequado nas cidades têm contribuído para a ocorrência de inundações e a conseqüente disseminação de doenças infecciosas. Adensamentos urbanos modernos são marcados por uma gigantesca rede de vias públicas que facilitam a circulação de bens e de pessoas, mas exigem a crescente utilização de asfalto e concreto que reduzem a capacidade de absorção d'água diretamente pelo solo, dificultando o escoamento de águas das chuvas pelas galerias pluviais ${ }^{5}$. Soma-se a esse aspecto o problema de destinação do lixo, com acondicionamento incorreto em terrenos baldios ou mesmo em espaços públicos. Em época de chuva, o lixo acumulado nas ruas é carregado até as galerias pluviais, entupindo-as. O solo impermeabilizado, incapaz de absorver as águas da chuva, aumenta o volume hídrico nas vias públicas favorecendo a ocorrência de inundações incontroláveis. Outra fonte de contaminação importante é o contato com a matéria orgânica úmida da lama resultante de inundações. As leptospiras sobrevivem no solo úmido por longos períodos, podendo infectar o indivíduo com a mesma facilidade que ocorre mediante contacto com água contaminada ${ }^{11}$

Nas áreas da cidade com altitudes mais baixas, potencialmente mais sujeitas às inundações, ocorreram $83,3 \pm 10 \%$ dos casos suspeitos e $79 \pm 11 \%$ dos confirmados. As populações assentadas ao longo de rios, sem tratamento de esgoto e sujeitos a enchentes, estão mais expostas à infecção por leptospiras.

Devido à carência de publicações relacionando a incidência de leptospirose com a distribuição espacial, sugerimos que casos da doença sejam, também, analisados utilizando-se recursos de geoprocessamento. Os cuidados de planejamento sanitário são importantes para proteger a saúde da população, evitando a transmissão de leptospiras e a ocorrência de epidemias de alto custo social.

O controle da população de roedores e da incidência da leptospirose exige fundamentalmente, um adequado manejo ambiental. As ações abrangem desde a simples manipulação e monitoramento do ambiente, como a limpeza de bueiros e a destinação adequada do lixo urbano, até a urbanização de favelas, drenagem e canalização de córregos e rios, prevesnindo a ocorrência de enchentes.

\section{AGRADECIMENTOS}

Os autores agradecem ao Diretor do Instituto Otávio Magalhães da Fundação Ezequiel Dias, Nery da Cunha Vital pelo material recebido para o estudo de geoprocessamento e ao Professor Ivan Barbosa Machado Sampaio do Departamento de Zootecnia da Universidade Federal de Minas Gerais pela análise estatística dos dados. 


\section{REFERÊNCIAS BIBLIOGRÁFICAS}

1. Andrade J, Brandão AP. Epidemiology of human leptospirosis, with special reference to greater Rio de Janeiro, Brazil, from 1970 to 1982. Memórias do Instituto Oswaldo Cruz 82: 91-100, 1987.

2. Correa MOA. Human leptospirosis in Brazil. International Journal of Zoonoses 2:1-9, 1975.

3. Cruz ML, Andrade J, Pereira MM. Leptospirosis in children in Rio de Janeiro. Revista da Sociedade Brasileira de Medicina Tropical 27: 5-9, 1994.

4. Faine S. Guideline for leptospirosis control. World Health Organization. Geneva, Offset publication, nr 67, p.161, 1982.

5. Franco RM. Enchentes, Meio Ambiente e Planejamento. Seminário sobre enchentes urbanas, Belo Horizonte, 1983. Anais de Eventos Técnicos. Fundação Centro Tecnológico de Minas Gerais/ CETEC, Belo Horizonte, p. 11-16, 1984.

6. Fundação Nacional de Saúde. Manual de Leptospirose. Ministério da Saúde, Brasília, 1995.

7. Gonçalves AJ, Carvalho JE, Guedes e Silva JB, Rozembaum R, Vieira AR. Hemoptysis and the adult respiratory distress syndrome as the causes of death in leptospirosis. Changes in the clinical and anatomicopathological patterns. Revista da Sociedade Brasileira de Medicina Tropical 25:261-270, 1992.

8. Horta CAC. Belo Horizonte: a construção de um saber geográfico. Dissertação de Mestrado, Universidade Federal de Santa Catarina, Florianópolis, 1994.

9. Johnson RC, Walby J, Henry RA, Auran RE. Cultivation of parasitic leptospires: effect of pyruvate. Applied Microbiology 26:118-119, 1973
10. Ko Al, Galvão RM, Ribeiro DCM, Johnson WDJr, Riley LW. Urban epidemic of severe leptospirosis in Brazil. Lancet 354:820-825, 1999.

11. Lomar AV, Veronesi R, Brito T, Diamont D. Leptospiroses. Veronesi R (ed) Doenças infecciosas e parasitárias 9a edição, Guanabara Koogan, Rio de Janeiro p. 987-1003, 1996.

12. Marotto PCF, Marotto MS, Santos DL, Souza TNL, Seguro AC Outcome of Leptospirosis in Children. American Journal Tropical Medical Hygiene 56: 307-310, 1997.

13. Moura ACM. Cartografia aplicada às análises urbanas. Caderno de Arquitetura e Urbanismo, Belo Horizonte 1, 1993.

14. Oliveira MAA, Chamone CB, Leocádio Filho G. Avaliação sorológica de leptospirose em Minas Gerais 1988-1993. Anais do 3ํㅡㄹ Encontro Nacional em Leptospirose. Fundação Oswaldo Cruz, Ministério da Saúde, Rio de Janeiro p.112, 1993.

15. Pereira RM. Leptospirose: alguns aspectos de seu comportamento epidemiológico no Estado de São Paulo, 19891994. Tese de Mestrado, Faculdade de Saúde Pública da Universidade de São Paulo, 1998.

16. Sakata EE, Yasuda PH, Romero EC, Silva MV, Lomar AV. Sorovares de Leptospira interrogans isolados de casos de leptospirose humana em São Paulo. Revista do Instituto de Medicina Tropical de São Paulo 34: 217-221, 1992.

17. Tavares Neto J, Andrade J, Hofer E, Oliveira GF, Couto Junior A Freqüência de aglutininas para leptospira, observadas em habitantes de Uberaba, Minas Gerais. Revista da Sociedade Brasileira de Medicina Tropical 29: 55-58,1996. 